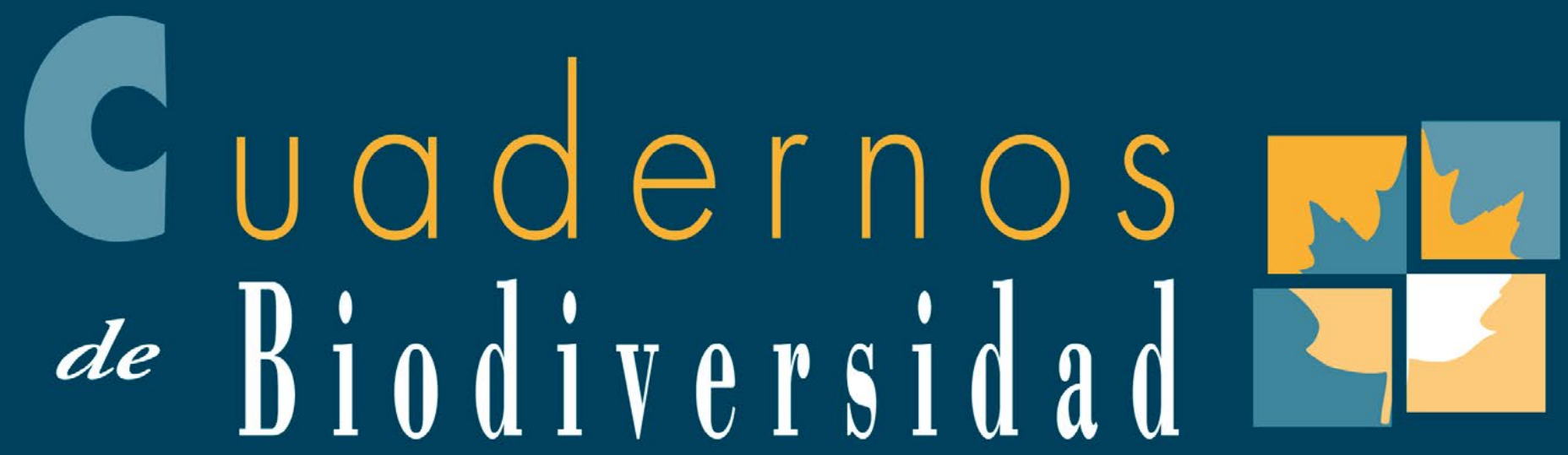

Cuadernos de biodiversidad número 48 • 2015
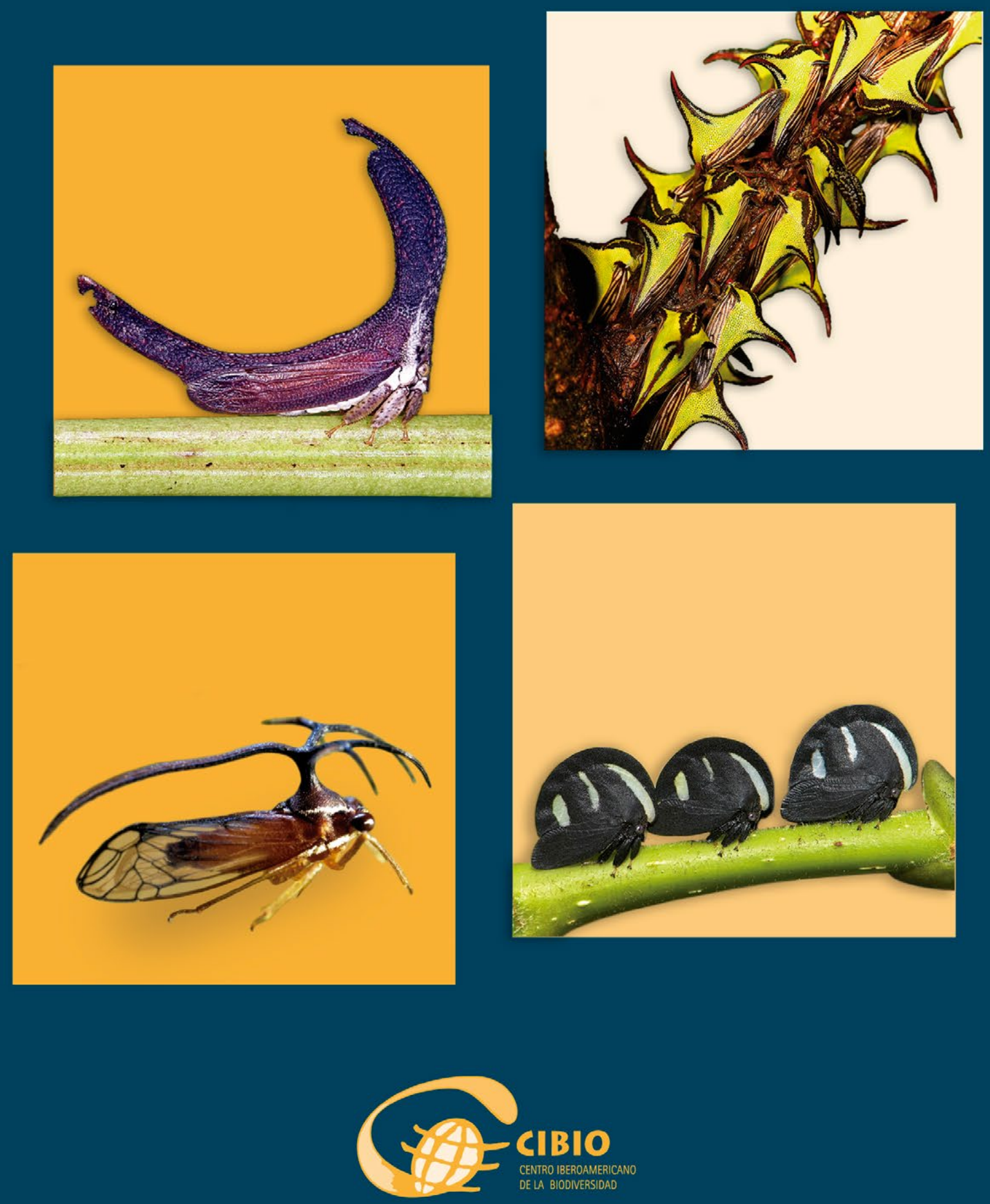

Universitat d'Alacant
Universidad de Alicante 


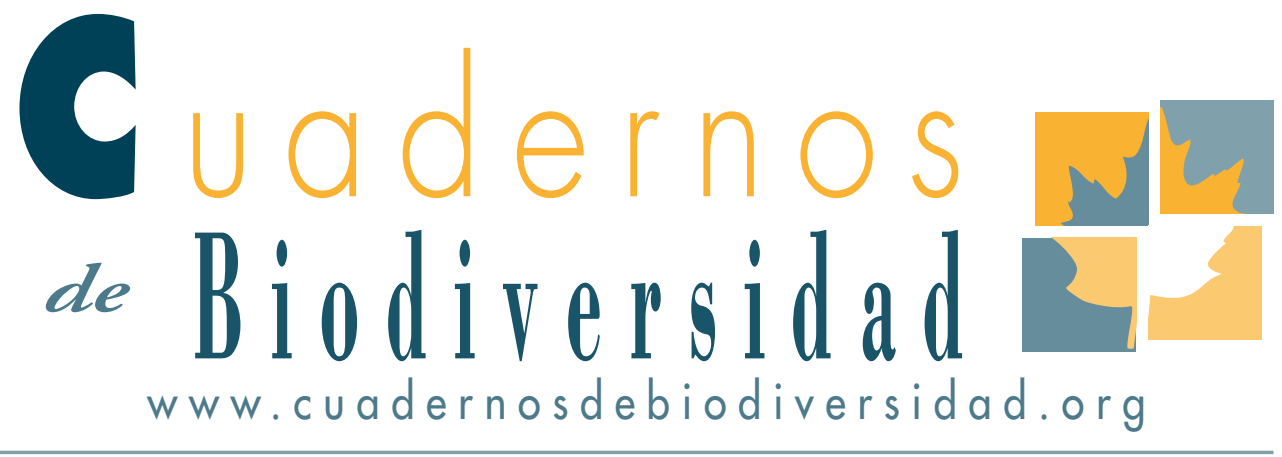

\section{Estrategias de conservación para Cołoneaster granatensis (Rosaceae), especie catalogada En Peligro de Extinción en la Comunitat Valenciana (España)}

\section{P. P. Ferrer-Gallego ${ }^{1,2^{*}}$, I. Ferrando ${ }^{1,2}$, F. Albert ${ }^{1,2}$, V. Martínez ${ }^{1,2}$, R. Carchano ${ }^{2}$, J. Pérez ${ }^{2}, R$. Herreros ${ }^{2}$, Xavier García Martí,} I. Francés ${ }^{2}, J$. V. Nieto ${ }^{2}, C$. Gisbert ${ }^{2}$, M. A. Bartolomé ${ }^{2}$, E. Martinez \& E. Laguna ${ }^{1}$

i Servicio de Vida Silvestre. Generalitat Valenciana. CiEF (Centro para la Investigación y Experimentación Forestal), Generalitat Valenciana, Av. Comarques del País Valencià, i i 4, E-46930, Quart de Poblet, Valencia, España.

2 VAERSA. Avda. Cortes Valencianas, no 20.460 i 5 , Valencia.

* FLORA.CIEF@GVA.ES

\section{ABSTRACT}

Cotoneaster granatensis (Rosaceae) is an endangered species in the Valencian Community with two natural populations known and 37 wild plants located in Alicante province. The strategy applied for conservation of this species comprises three steps in a cyclical method: in situ (first step) - ex situ - in situ (second step). Current conservation status for this species has been evaluated as a part of the first step in situ actions. Ex situ conservation activities consisted of germplasm conservation on seed banks, creation and maintenance of collections of livings plants and production of new plants in order to develop reintroductions (in situ actions: second step) in natural populations. The seed germination is a complex issue, but an effective protocol has been established reaching values over $60 \%$ of germination. The plant propagation by cuttings has not been successful. In situ actions such as reducing the threat of herbivory 
has been also essential to enhance natural populations. The initial results on plantations in the natural habitat show high survival rates of specimens.

Key words: Falso membrillo, endangered species, seeds, conservation translocation.

\section{INTRODUCCIÓN}

La guillomera o falso membrillo (Cotoneaster granatensis Boiss.) es una especie de la familia de las rosáceas, catalogada como "En peligro de extinción" en la Comunidad Valenciana (Decreto 70/2009; Orden 6/2013) (véase Anónimo, 2009, 2013; Aguilella et al., 2009), e incluida en el Programa Valenciano de Conservación de Recursos Genéticos Forestales (ECOGEN) (Prada et al., 2012). Resulta una planta endémica de la Península Ibérica, exclusiva de las sierras béticas, cuyo límite de distribución septentrional está en la Comunidad Valenciana, concretamente en los núcleos poblacionales localizados en la cumbre de la Sierra de Aitana (Alicante).

En el territorio valenciano, hasta el momento, tan sólo se conocen dos núcleos poblacionales (unidades de seguimiento a efectos de gestión): 1) Font de la Forata-Passet de la Rabosa (términos municiaples de Benifato y Confrides) y 2) Moleta d'Aitana (Benifato), creciendo en ambos casos en ambientes frescos y rupestres, pedregales junto al pie de paredones calizos, formando parte de la vegetación espinosa y de orlas forestales por encima de los $900 \mathrm{~m}$ de altitud. Desde el punto de vista de su estado de conservación en la Comunidad Valenciana, estos dos núcleos poblacionales están sujetos a varias amenazas, siendo una de las principales el intenso ramoneo por parte del ganado doméstico y de otros herbívoros introducidos recientemente en el territorio, como por ejemplo el arruí o el muflón (Aguilella et al., 2009). Esta actividad parece ser la responsable de la ausencia de regeneración natural, debido a la predación que sufren los individuos jóvenes, y la depauperación de ramaje y follaje de los individuos adultos, de modo que apenas llegan a producir flores y frutos. Por otra parte, tanto los incendios y muy probablemente los desprendimientos rocosos, constituyen amenazas adicionales para la especie.
Los objetivos planteados para la conservación de esta planta en la Comunidad Valenciana han consistido en la caracterización de los núcleos poblacionales o unidades de seguimiento mediante censos exhaustivos y la georreferenciación de los individuos, así como la búsqueda de nuevos ejemplares. Además, se evalúa el efecto de la instalación de vallados de exclusión en la producción de frutos y en el crecimiento y vigor de algunos individuos. Por otra parte, se analiza cuantitativa y cualitativamente el material vegetal de propagación (semillas y esquejes o estaquillas), la producción de planta a partir de la colección mantenida ex situ en el CIEF (Centro para la Investigación y Experimentación Forestal de la Generalitat Valenciana) y el éxito de implantación de ejemplares introducidos in situ.

\section{MATERIAL Y MÉTODOS}

El método de trabajo con esta especie sigue el modelo cíclico propuesto por Ferrer-Gallego et al. (2013) desarrollando una estrategia basada en tres fases; in situ (de primer paso) - ex situ - in situ (de segundo paso). Los trabajos in situ (de primer paso) se centran en el diagnóstico y situación real de la especie en el hábitat natural (detección de amenazas, censos, georreferenciación, búsqueda de nuevos ejemplares, etc.); las actividades ex situ están encaminadas a la conservación de germoplasma (semillas), multiplicación y producción de ejemplares mediante su cultivo, creación de colecciones de planta viva para abastecimiento de germoplasma, etc.; y las acciones in situ (de segundo paso) contemplan los trabajos activos y directos desarrollados en los núcleos poblacionales naturales, refuerzo poblacional, creación de neopoblaciones, vallados de exclusión, restauración de hábitat, etc.

\section{In situ (de primer paso)}

Cada núcleo poblacional se ha considerado como una unidad de seguimiento a efectos demográficos. Hasta el año 2012 no habían sido individualizados estos dos núcleos diferentes para la trazabilidad del germoplasma conservado en el banco. El último censo demográfico de los ejemplares silvestres se realizó en 2013 mediante censo directo, tomando 
las coordenadas UTM (Universal Transversal Mercator) para la georreferenciación de los individuos en ETRS89 (European Terrestrial Reference System 1989), con una precisión de 1 metro. El análisis y representación cartográfica de la información se ha realizado utilizando el software libre gvSIG (v. 1.1.2). El censo de las unidades de seguimiento también contempló la caracterización de los ejemplares según la tipificación de adulto-reproductor o juvenil, y en el caso de la población de Font de la Forata-Passet de la Rabosa se tomaron datos de altura y diámetro máximo de los individuos.

\section{Ex situ}

Para el estudio, caracterización, procesado y conservación de las accesiones de germoplasma (semillas y material vegetativo de reproducción mediante esquejado) se ha seguido la metodología expuesta por Ferrer-Gallego et al. (2013). Los trabajos se han realizado en las instalaciones del CIEF, cuyas colecciones de germoplasma están detalladas en este mismo trabajo de Ferrer-Gallego et al. (2013). La viabilidad ha sido medida como el porcentaje de semillas bien formadas (viables) frente a las vacías del total del lote, mediante flotación en agua. Para las accesiones anteriores a 2012, sólo se calcularon viabilidades para aquellas accesiones que superaran la cantidad de 150 semillas. Para el estudio de la germinación se ha seleccionado una muestra al azar de 200 semillas de la accesión divididas en 4 réplicas de 50 semillas/placa. Antes de la siembra, las semillas fueron sometidas a un pretratamiento de escarificación ácida con ácido sulfúrico (H2SO4 durante 1 hora), seguido de una imbibición en giberelinas (GA3 500 ppm) durante 24 horas y una estratificación en frío a $4^{\circ} \mathrm{C}$ en placas de Petri con agar $0.6 \%$ o papel de filtro durante 30 días. Pasado este tiempo las semillas se introdujeron en cámara germinadora en condiciones de $15^{\circ} \mathrm{C}, 24$ horas oscuridad. La lectura de la germinación fue diaria y a medida que ocurría la germinación se procedía al repicado de las plántulas a contenedores de tipo QP 35T de $200 \mathrm{~cm} 3$ en primera instancia y repicados a contenedores de 4 litros (tipo forestal) con sustrato compuesto por turba:fibra de coco:perlita (3:1:1) al alcanzar la primera savia. Los ensayos de germinación se dieron por concluidos tras un mes sin que ninguna germinación tuviese lugar. En ese momento se realizó la prueba de corte a las semillas no germinadas evaluando la porción de semillas frescas (viables), vacías, muertas o no bien conformadas.

La multiplicación vegetativa mediante esquejado se hizo en mesa de cultivo con sustrato de turba y perlita a partes iguales y temperatura de $25^{\circ} \mathrm{C}$ constantes $24 \mathrm{~h}$. Las estacas fueron recolectadas de los ejemplares más sanos y vigorosos de los núcleos poblacionales naturales en dos fases, una durante otońo de 2010 y otra en otońo-invierno de 20112012. En ambas se recolectaron esquejes de varios individuos y de diferentes tamaños de calibre (entre 0,5-2 cm de diámetro) con un tamaño de $25 \mathrm{~cm}$ de longitud. Para su cultivo se practicó un corte en flauta $\left(45^{\circ}\right)$ en la parte superior y recto $\left(90^{\circ}\right)$ en la inferior. Todas las estacas fueron sumergidas en una dilución de ácido indolbutílico (IBA 0,2\% ppm), naftilacético (ANA 0,2\% ppm) y fungicida ZIRAM (4\% ppm) durante 20 segundos y después se lavaron con agua destilada.

\section{In situ (de segundo paso)}

Los refuerzos poblacionales se han realizado en todos los casos con planta cultivada a partir de material de procedencia local de los núcleos poblacionales ya indicados y según las recomendaciones de la IUCN/SSC (2013). Los ejemplares introducidos se han obtenido a partir de la germinación de semillas y cultivo de plantas en condiciones de vivero en las instalaciones del CIEF, según lo indicado en el apartado anterior. Dichos ejemplares tienen en todos los casos 1-2 savias.

Los primeros trabajos de introducción de planta en campo se han realizado en dos fases, una primera en enero de 2014, con un total de 50 ejemplares plantados, y una segunda en diciembre del mismo año, con un total de 53 ejemplares plantados. Todos los ejemplares fueron introducidos dentro de la microrreserva de flora denomina Coll del Ventisquer, en el término de Cofrides (Anónimo, 2010), en la cumbre de Aitana, al pie de paredones rocosos y junto a la orla espinosa de vegetación. Se ha utilizado en todos los casos protectores de malla metálica para salvaguardar a los ejemplares plantados 
(enrejado de malla cuadrada de $10 \mathrm{~mm}$ de luz). No se han realizado riegos de apoyo en las plantaciones.

Los vallados de exclusión para ungulados se han instalado en los dos núcleos. El tipo de malla empleado ha sido metálico (malla de gallinero), con enrejado hexagonal de $5 \mathrm{~mm}$ de luz. En Moleta d'Aitana se han creado dos cercados, uno para proteger a un ejemplar adulto y otro que encierra a dos ejemplares, uno de ellos también adulto; mientras que en la población de Passet Rabosa-Font de la Forata se han instalado 3 cercados que protegen varios ejemplares juveniles y también de pequeña talla pero reproductores.

\section{RESULTADOS Y DISCUSIÓN}

\section{Conservación in situ (de primer paso)}

Para la unidad de seguimiento de Font de la Forata-Passet de la Rabosa (Benifato-Confrides) se contabilizó un total de 27 ejemplares, repartidos en un área de $15.055 \mathrm{~m} 2$. La estructura por clases de edad indica un total de 10 adultos-reproductores y 17 juveniles (Figuras 1 y 2b). En la unidad de seguimiento de Moleta d'Aitana (Benifato) se han censado un total de 10 individuos, 8 ejemplares adultos-reproductores y 2 juveniles, ocupando un área de $47.338 \mathrm{~m} 2$ (Figura 2a).
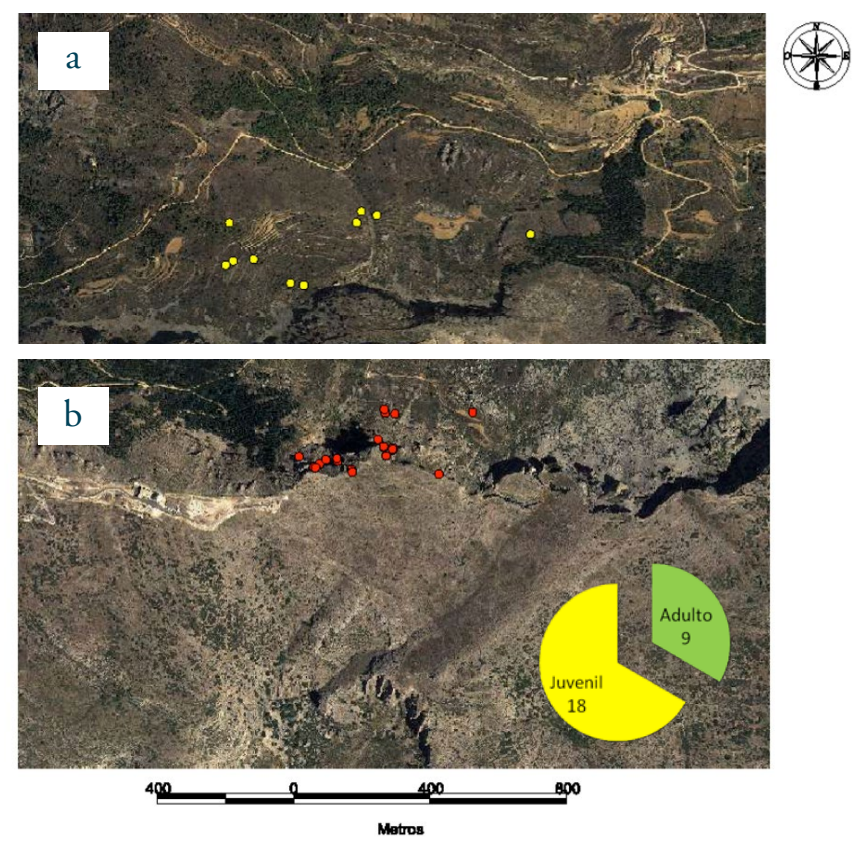

Figura 2. Cartografía de la subpoblación Moleta d'Aitana (a) y Passet de la Rabosa-Font de la Forata (b); gráfico: identificación del estado de madurez de los ejemplares e imagen del hábitat donde vive la especie.

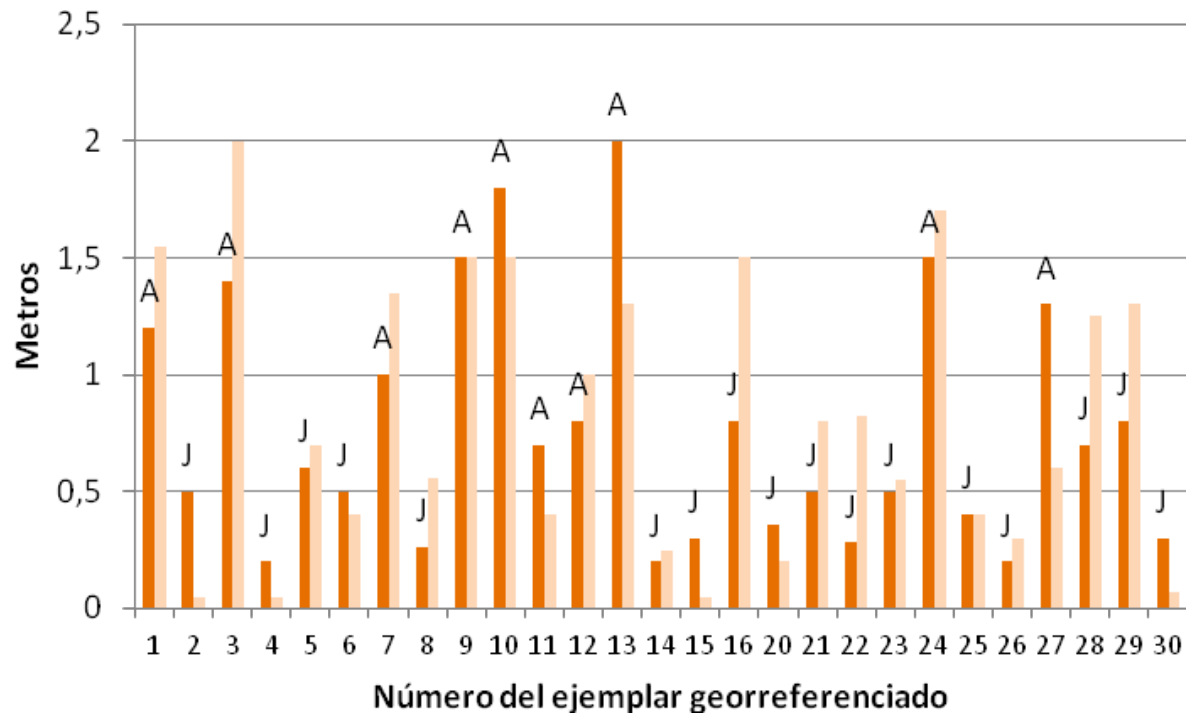

Figura 1. Caracterización de la subpoblación Font de la Forata-Passet de la Rabosa. Distribución del tamaño de los ejemplares según su altura (naranja) y su diámetro máximo (marrón claro). A) Adulto; J) Juvenil. Nota: la gráfica muestra un total de 27 ejemplares aunque en el eje de las $\mathrm{x}$ aparece el número de referencia de los individuos, ya que los ejemplares no 17,18 y 19 han causado baja recientemente. 


\section{Conservación ex situ}

\section{Accesiones}

En la actualidad se conservan 9 accesiones en la colección de base del banco de germoplasma del CIEF, a $-20^{\circ} \mathrm{C}$, que se corresponden con 4 años de recolección, y 11 accesiones en la colección activa a $4^{\circ} \mathrm{C}$, realizadas entre 2012 y 2013 , de las cuales 6 contienen semillas de la subpoblación de Font de la Forata- Passet de la Rabosa y 5 de Moleta d'Aitana. En la colección de base, las accesiones contienen un total de 3.521 semillas pertenecientes a los dos núcleos poblacionales conocidos. En la colección activa el número total de semillas conservadas es 6.217 , con una viabilidad de las accesiones entre el $44 \%$ al $75 \%$ (Tabla 3).

Tabla 3. Accesiones conservadas de $C$. granatensis en el banco de germoplasma del CIEF. $\left(^{*}\right)$ sin datos; $(\dagger)$ hasta el año 2012 las recolecciones no fueron identificadas como pertenecientes a dos núcleos poblacionales o unidades de seguimiento diferentes.

\begin{tabular}{|c|c|c|c|c|c|}
\hline \multicolumn{6}{|c|}{ Colección base conservada a $-20^{\circ} \mathrm{C}$} \\
\hline Código accesión & $\begin{array}{l}\text { Unidad de } \\
\text { seguimiento }\end{array}$ & $\begin{array}{l}\text { Fecha recolec- } \\
\text { ción }\end{array}$ & Peso $(\mathrm{g})$ & No semillas & $\%$ Viabilidad \\
\hline A13820100DA & \multirow{9}{*}{$\begin{array}{c}\text { Forata- Passet + } \\
\text { Moleta d'Aitana } \\
\qquad(\dagger)\end{array}$} & $01 / 09 / 2000$ & 2,091 & 100 & * \\
\hline A13820109DA & & 01/09/2009 & 13,506 & 646 & 67 \\
\hline A13820209DA & & $28 / 08 / 2009$ & 3,596 & 172 & 48 \\
\hline A13820310DA & & $30 / 09 / 2010$ & 2,342 & 112 & * \\
\hline A13820410DA & & $30 / 09 / 2010$ & 1,777 & 88 & * \\
\hline A13820510DA & & $30 / 09 / 2010$ & 0,101 & 5 & * \\
\hline A13820610DA & & $31 / 08 / 2010$ & 4,038 & 200 & 35 \\
\hline A13820311DA & & $31 / 08 / 2011$ & 0,444 & 22 & * \\
\hline $\mathrm{A} 13820411 \mathrm{DA}$ & & $31 / 08 / 2011$ & 0,302 & 15 & $*$ \\
\hline \multicolumn{6}{|c|}{ Colección activa conservada a $4^{\circ} \mathrm{C}$} \\
\hline A185B & Forata- Passet & $24 / 09 / 2012$ & 7,422 & 233 & 75 \\
\hline A185C & Forata- Passet & $24 / 09 / 2012$ & 6,914 & 155 & 44 \\
\hline A185D & Moleta d'Aitana & $26 / 10 / 2012$ & 26,810 & 1.196 & 68 \\
\hline A185E & Moleta d'Aitana & $26 / 10 / 2012$ & 52,975 & 1.491 & 45 \\
\hline $\mathrm{A} 185 \mathrm{~F}$ & Moleta d'Aitana & $26 / 10 / 2012$ & 15,076 & 446 & 57 \\
\hline A185I & Forata- Passet & $22 / 08 / 2013$ & 13,574 & 555 & 54 \\
\hline A185J & Forata- Passet & $22 / 08 / 2013$ & 0,854 & 40 & 65 \\
\hline $\mathrm{A} 185 \mathrm{G}$ & Moleta d'Aitana & $04 / 10 / 2013$ & 3,115 & 100 & 73 \\
\hline $\mathrm{A} 185 \mathrm{M}$ & Forata- Passet & $04 / 10 / 2013$ & 22,001 & 557 & 52 \\
\hline A185N & Moleta d'Aitana & $04 / 10 / 2013$ & 20,996 & 698 & 67 \\
\hline A185L & Forata- Passet & $18 / 09 / 2013$ & 15,428 & 746 & 65 \\
\hline
\end{tabular}


Tabla 4. Caracterización morfológica de los frutos y semillas de Cotoneaster granatensis.

\begin{tabular}{l|l|l}
\multicolumn{1}{c|}{ Parámetros } & \multicolumn{1}{c|}{ Frutos } & \multicolumn{1}{c}{ Semillas } \\
\hline $\begin{array}{l}\text { Dimensiones }(\mathbf{L ~} \mathbf{~ m m} \mathbf{x} \text { A } \\
\mathbf{m m})(\mathbf{X} \pm \text { s.d.) }\end{array}$ & $7,356 \pm 0,619 \times 7,783 \pm 0,550$ & $5,876 \pm 0,289 \times 4,383 \pm 0,278$ \\
\hline Color y forma & $\begin{array}{l}\text { rojo brillante en estado maduro, con } \\
\text { forma globosa o piriforme }\end{array}$ & $\begin{array}{l}\text { marrón, obovadas, con una cara lisa } \\
\text { y otra con crestas longitudinales }\end{array}$ \\
\hline $\begin{array}{l}\text { Peso fresco (g) de } \mathbf{1 0 0} \\
\text { unidades }(\mathbf{X} \pm \text { s.d.) }\end{array}$ & $35,594 \pm 20,364$ & $2,091 \pm 0.315$
\end{tabular}

\section{VIABILIDAD DE LAS SEMILLAS Y ENSAYOS DE GERMINACIÓN}

La viabilidad de las accesiones (semillas no vacías) muestra valores entre el 44\% y el 75\% (Tabla 3). Con el protocolo indicado en el apartado de material y métodos se ha obtenido un valor de germinación del $66,10 \pm 5,16 \%$ para la accesión que muestra el mayor valor de viabilidad (75\%) y que corresponde al código A185B (véase Tabla 3). (Figura 3).
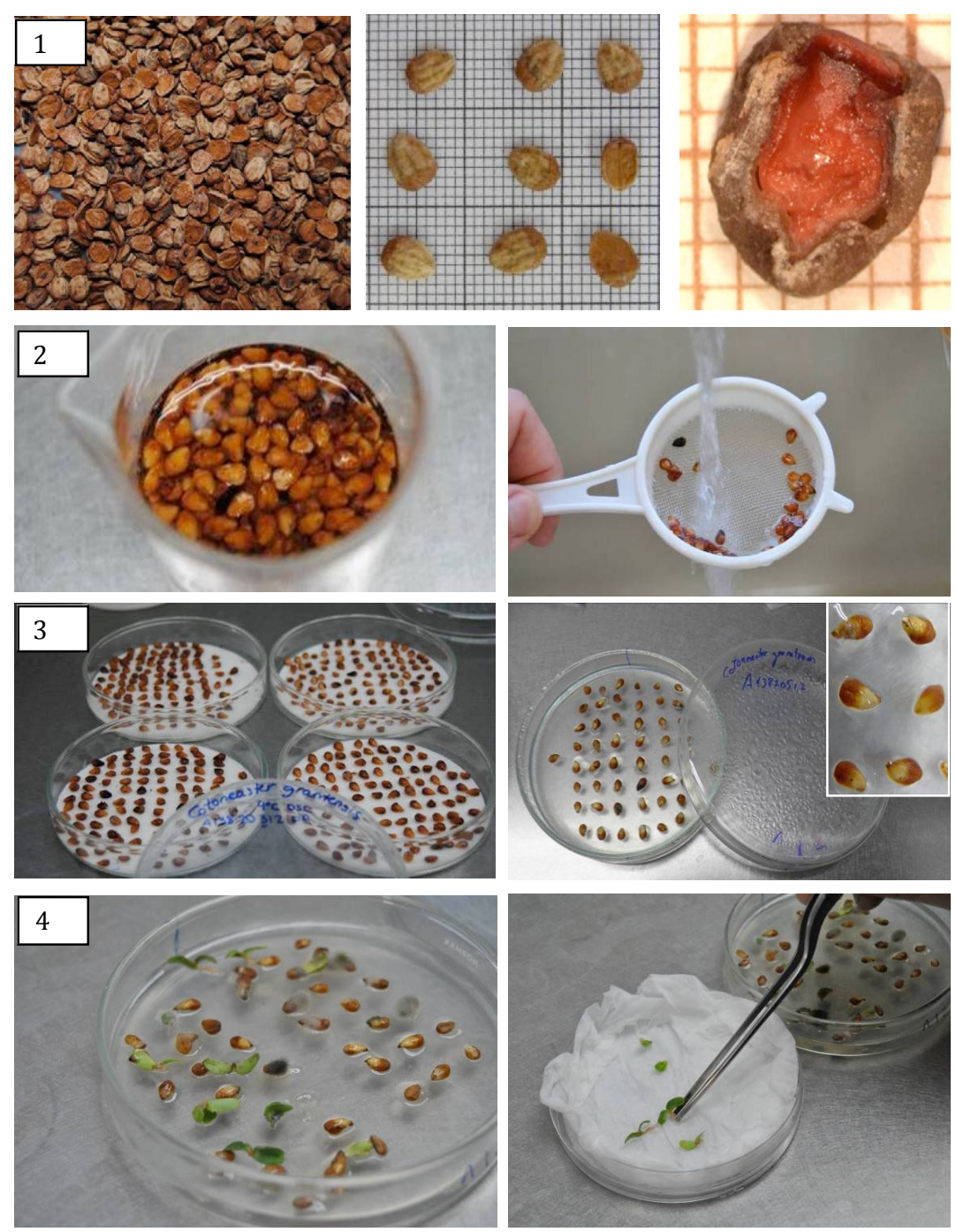

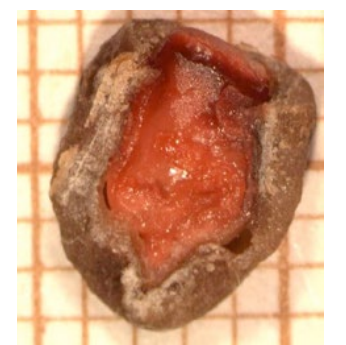

Figura 3. Tipo de semilla de Cotoneaster granatensis y protocolo de germinación: 1) caracterización morfológica y estudio de vialidad aparente de la semilla; 2) tratamiento con ácido sulfúrico para escarificar y hacer permeable la cubierta; 3 ) placas de Petri con papel de filtro y agar-agar para las siembras de semillas; 4) detalle de la germinación y repicado para pasar a sustrato. 


\section{CULTIVO}

La producción de planta ex situ en las instalaciones del CIEF tuvo dos finalidades principales. Por un lado, parte de los ejemplares se destinaron a la creación de una colección de planta viva (huerto semillero) para la multiplicación de germoplasma (semillas y estaquillas). Este huerto está compuesto actualmente por un total de 20 ejemplares y representa al 50\% los dos núcleos poblacionales hasta ahora conocidos (véase Figura 5). Por otra parte, el resto de la producción se cultivó con el objetivo de realizar los trabajos de restitución en el hábitat natural.
Las dos líneas ensayadas de producción de planta fueron: 1) esquejado a partir de material de los ejemplares silvestres y adultos donantes de la colección $e x$ situ del CIEF (Figura 4), y 2) cultivo de las plántulas obtenidas por germinación de las semillas recolectadas de los ejemplares silvestres y de las plantas madre del huerto productor. Los resultados obtenidos mediante la primera técnica no han sido en ningún caso exitosos, a pesar de haberse observado brotes vegetativos durante las primeras semanas, no se alcanzó buena rizogénesis y los esquejes no lograron enraizar adecuadamente.

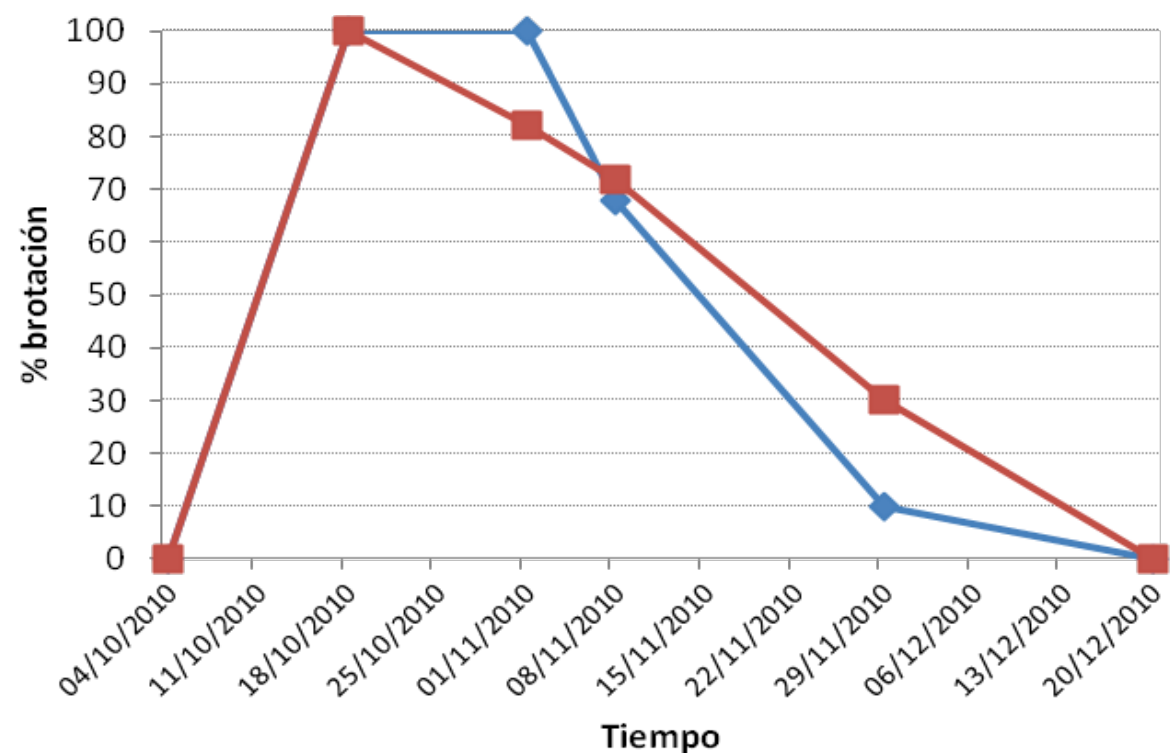

Figura 4. Porcentaje de brotación a lo largo del tiempo de los esquejes realizados para dos accesiones de germoplasma (línea roja: accesión A13820110DA; azul: A13820210DA).

Respecto a la producción de planta a partir de la germinación, se consiguieron 145 plantas, en una primera fase de establecimiento de protocolos de cultivo, que se utilizaron para los trabajos de reintroducción y la creación del huerto semillero. En la actualidad, mediante el protocolo de germinación establecido indicado anteriormente se mantienen en cultivo aproximadamente 1000 plantones (Figura 5). 

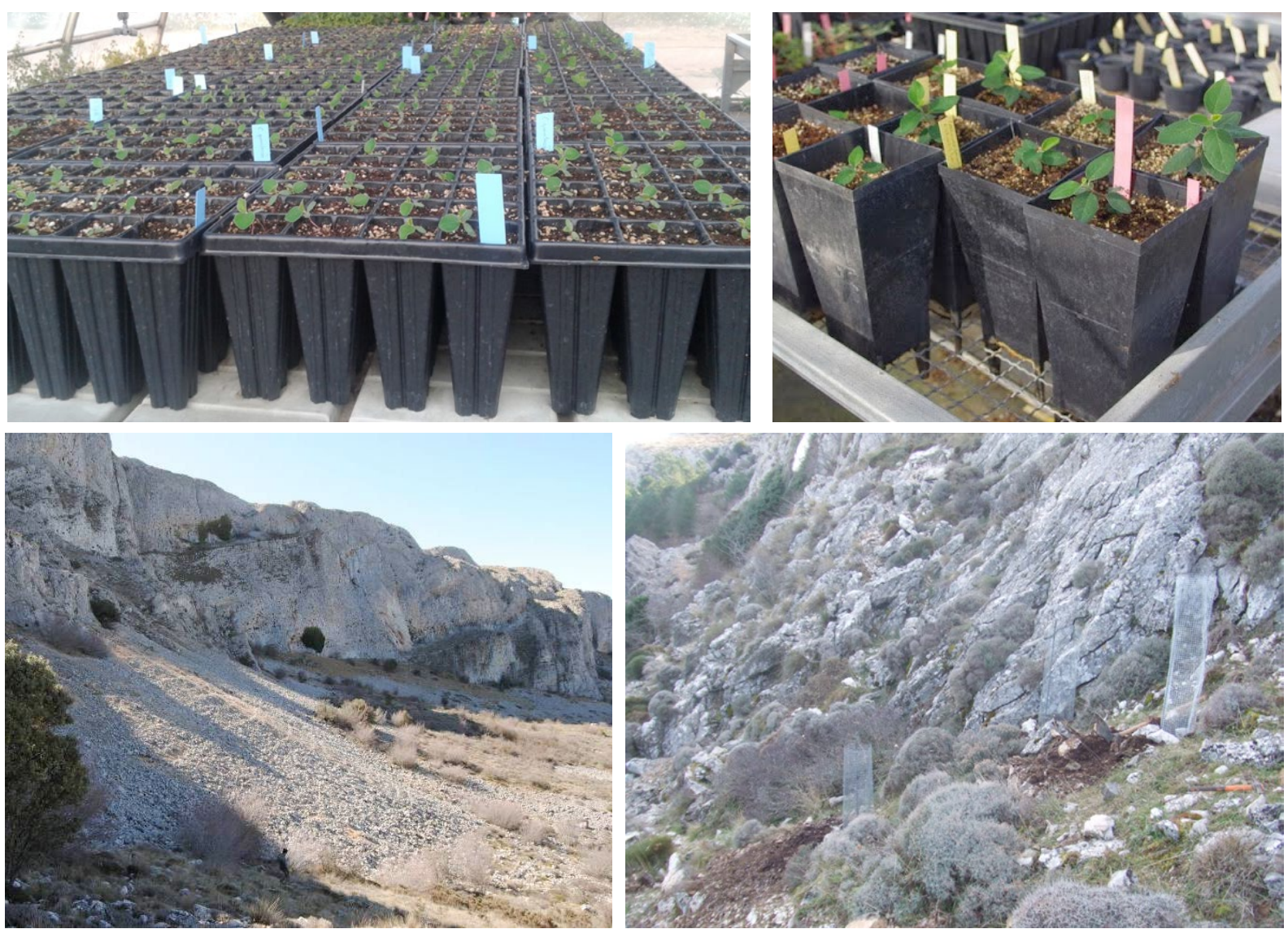

Figura 5. Producción de planta de Cotoneaster granatensis a partir de la germinación de las semillas (arriba); hábitat de la especies en la Sierra de Aitana plantación de ejemplares en la microrreserva de Coll del Ventisquer (abajo).

\section{Conservación in situ (de segundo paso)}

\section{Vallados de exclusión}

Hasta 2011, la cantidad de semilla producida, en su mayoría recolectada para las experiencias aquí indicadas, era relativamente baja, como ilustran los datos de la Tabla 3.

En 2011 se instalaron vallados de exclusión para ejemplares adultos-reproductores en la población de Moleta d'Aitana (Figura 6) y un grupo de varios ejemplares juveniles en la población de Passet Rabosa-Font de la Forata. Esta acción ha permitido a las plantas protegidas crecer y alcanzar un mayor vigor, produciendo al tiempo una gran cantidad de semillas y de muy buena calidad en el caso de la población de Moleta d'Aitana. En total la cantidad de semillas recolectada en los ejemplares cercados ha sido 2.687 en 2012, lo que supone el $86 \%$ de todo el germoplasma recolectado en 2012 para la población de Moleta d'Aitana y el 76\% para la suma de los dos núcleos poblacionales (Tabla 3).

\section{Plantaciones en campo}

El primer censo realizado en febrero de 2015 sobre los 50 ejemplares plantados en enero de 2014 muestra un valor del $92 \%$ de supervivencia; tan solo 4 ejemplares no han logrado instalarse, un porcentaje muy bajo si se tiene en cuenta la prolongada sequía acaecida durante 2014. 

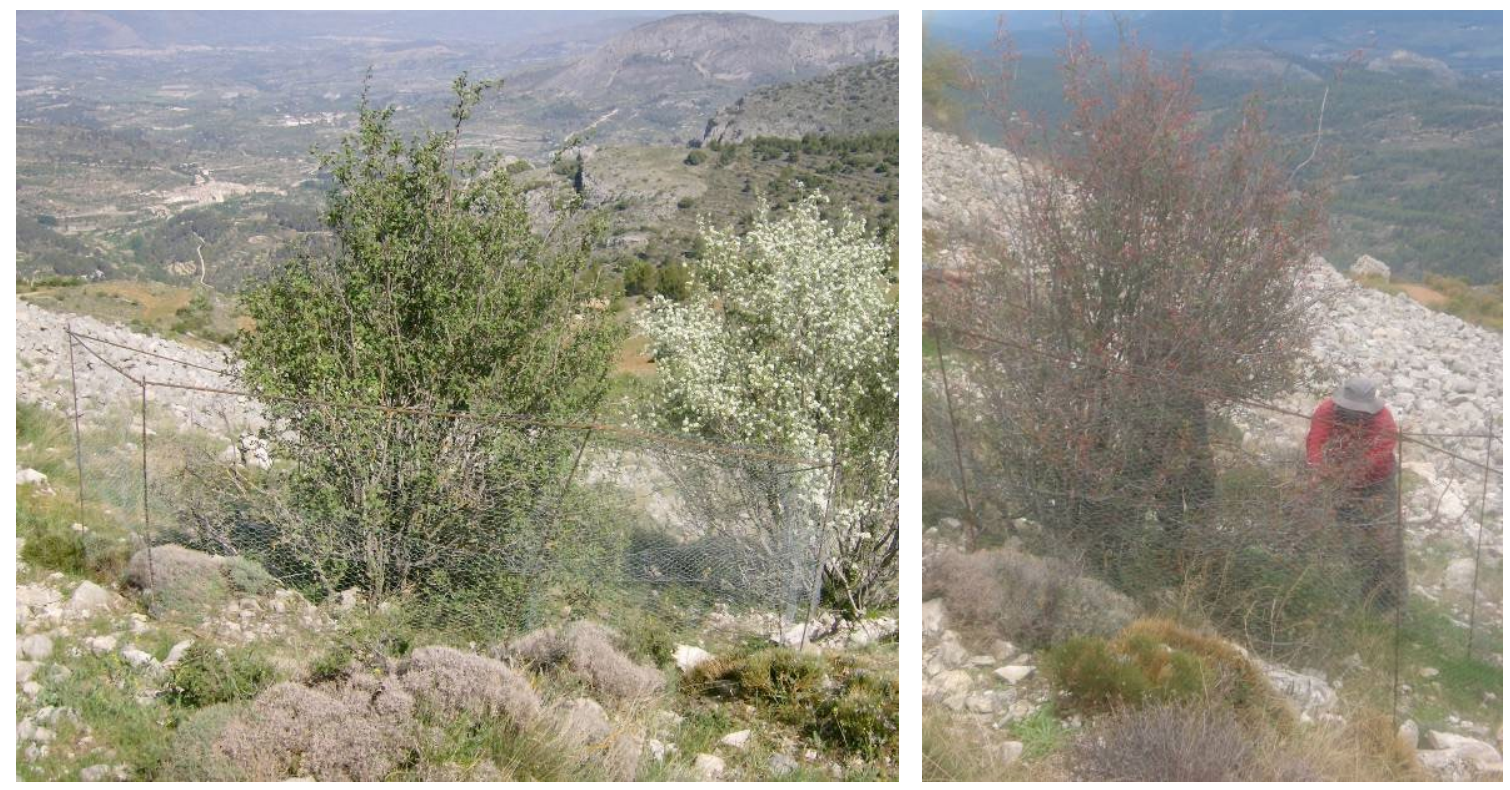

Figura 6. Vallado de exclusión practicado en un ejemplar de Cotoneaster granatensis en la población de Moleta d'Aitana y aspecto de la planta en el momento de la maduración y recolección de los frutos.

\section{DISCUSIÓN Y CONCLUSIONES}

El vallado perimetral de exclusión ha favorecido la producción de frutos y semillas, y se muestra como una práctica recomendable para asegurar la producción y el crecimiento de los individuos mientras continúe la presencia de ungulados en la zona.

Las pruebas de producción mediante estaquillado no han dado buenos resultados. Debido fundamentalmente a la falta de buenos ejemplares donantes dado el debilitamiento de las plantas por el efecto de la herbívora.

La viabilidad de las semillas es bastante alta, sin embargo, conseguir germinarlas en laboratorio es complejo, entre otras causas, debido al grosor de la cubierta externa (exo y mesocarpo) y la dormición fisiológica y estructural que presentan. No obstante, se ha podido establecer un protocolo que logra obtener resultados superiores al 60\% de germinación.

La supervivencia de los ejemplares introducidos en campo alcanza valores altos en una primera de evaluación, transcurrido algo más de un año desde la primera plantación.
Consideramos de gran importancia para la conservación de esta especie seguir con los trabajos de recolección de semilla, producción de ejemplares en vivero y posteriores trabajos de restitución. En este sentido, las plantaciones deberían ir destinadas a reforzar los núcleos poblacionales existentes, como por ejemplo la depauperada Moleta d'Aitana, pero sobre todo a crear nuevas poblaciones. Nuevas poblaciones podrían crearse tanto en la Sierra de Aitana como en otros enclaves próximos, donde no existan las amenazas que ahora soporta en esta sierra, y que reúnen condiciones ambientales adecuadas para albergar la especie, como por ejemplo la cima del Puig Campana o la Sierra de Mariola, lo que aumentaría el área de distribución de la planta en el territorio valenciano.

\section{AGRADECIMIENTOS}

Gracias a nuestros compañeros del CIEF y del Servicio de Vida Silvestre de la Generalitat Valenciana por su colaboración en los diferentes trabajos que trata esta comunicación. Las actividades aquí indicadas han sido cofinanciadas por la Comisión Europea a través del programa operativo de los fondos FEOGAOrientación y FEADER en la Comunidad Valenciana. 


\section{REFERENCIAS}

Aguilella, A., Fos, S. \& Laguna, E. (eds.). 2009. Catálogo Valenciano de Especies de Flora Amenazadas. Colección Biodiversidad, 18. Conselleria de Medi Ambient, Aigua, Urbanisme i Habitatge, Generalitat Valenciana. Valencia. $358 \mathrm{pp}$.

Anónimo 2009. Decreto 70/2009, de 22 de mayo, del Consell, por el que se crea y regula el Catálogo Valenciano de Especies de Flora Amenazadas y se regulan medidas adicionales de conservanción. Diari Oficial de la Comunitat Valenciana 6021: 20143-20162.

Anónimo 2013. Orden 11/2010, de 26 de mayo, de la Conselleria de Medio Ambiente, Agua, Urbanismo y Vivienda. Diari Oficial de la Comunitat Valenciana 6286: 23253-23279.

Anónimo 2013. Orden 6/2013, de 25 de marzo, de la Conselleria de Infraestructuras, Territorio y Medio Ambiente, por la que se modifican los listados valencianos de especies protegidas de flora y fauna. Diari Oficial de la Comunitat Valenciana 6996: 8682-8690.
Ferrer-Gallego, P.P., Ferrando, I., Gago, C. \& Laguna, E. (eds.) 2013. Manual para la conservación de germoplasma y el cultivo de la flora valenciana amenazada. Colección Manuales Técnicos Biodiversidad, 3. Conselleria d'Infraestructures, Territori i Medi Ambient. Generalitat Valenciana. Valencia. 252 pp.

Prada, M.A. Herreros, R. Ortiz, G. \& Marzo, M. 2012. ECOGEN. Programa Valenciano de Conservación de Recursos Genéticos Forestales. 2011-2020. Conselleria de Infraestructuras, Territorio y Medio Ambiente. Generalitat Valenciana. 49 pp.

IUCN/SSC 2013. Guidelines for Reintroductions and Other Conservation Translocations. Version 1.0. Gland, Switzerland: IUCN Species Survival Commission, viiii + 57 pp. 